Aurélio Campilho · Mohamed Kamel (Eds.)

\title{
Image Analysis and Recognition
}

11th International Conference, ICIAR 2014 Vilamoura, Portugal, October 22-24, 2014 Proceedings, Part I 


\section{Editors}

Aurélio Campilho

Faculty of Engineering

University of Porto

Porto

Portugal

\author{
Mohamed Kamel \\ Department of Electrical and Computer \\ Engineering \\ University of Waterloo \\ Waterloo, ON \\ Canada
}

ISSN 0302-9743

ISBN 978-3-319-11757-7

DOI 10.1007/978-3-319-11758-4

ISSN 1611-3349 (electronic)

ISBN 978-3-319-11758-4 (eBook)

\title{
Library of Congress Control Number: 2014950801
}

LNCS Sublibrary: SL6 - Image Processing, Computer Vision, Pattern Recognition, and Graphics

\author{
Springer Cham Heidelberg New York Dordrecht London
}

(C) Springer International Publishing Switzerland 2014

This work is subject to copyright. All rights are reserved by the Publisher, whether the whole or part of the material is concerned, specifically the rights of translation, reprinting, reuse of illustrations, recitation, broadcasting, reproduction on microfilms or in any other physical way, and transmission or information storage and retrieval, electronic adaptation, computer software, or by similar or dissimilar methodology now known or hereafter developed. Exempted from this legal reservation are brief excerpts in connection with reviews or scholarly analysis or material supplied specifically for the purpose of being entered and executed on a computer system, for exclusive use by the purchaser of the work. Duplication of this publication or parts thereof is permitted only under the provisions of the Copyright Law of the Publisher's location, in its current version, and permission for use must always be obtained from Springer. Permissions for use may be obtained through RightsLink at the Copyright Clearance Center. Violations are liable to prosecution under the respective Copyright Law.

The use of general descriptive names, registered names, trademarks, service marks, etc. in this publication does not imply, even in the absence of a specific statement, that such names are exempt from the relevant protective laws and regulations and therefore free for general use.

While the advice and information in this book are believed to be true and accurate at the date of publication, neither the authors nor the editors nor the publisher can accept any legal responsibility for any errors or omissions that may be made. The publisher makes no warranty, express or implied, with respect to the material contained herein.

Printed on acid-free paper

Springer is part of Springer Science+Business Media (www.springer.com) 
Face Image Super-Resolution Based on Topology ICA and Sparse

Representation ...........................

Yongtao Liu, Hua Yan, Xiushan Nie, and Zhen Liu

Iterative Sparse Coding for Colorization Based Compression

Suk-Ho Lee, Paul Oh, and Moon Gi Kang

Noise Modelling in Parallel Magnetic Resonance Imaging:

A Variational Approach. . . . . . . . . . . . . . . . . . . . .

Adrián Martín and Emanuele Schiavi

\section{Image Restoration and Enhancement}

An Examination of Several Methods of Hyperspectral Image Denoising:

Over Channels, Spectral Functions and Both Domains . . . . . . . . . . .

Daniel Otero, Oleg V. Michailovich, and Edward R. Vrscay

Towards a Comprehensive Evaluation of Ultrasound Speckle Reduction . . . . Fernando C. Monteiro, José Rufino, and Vasco Cadavez.

An Evaluation of Potential Functions for Regularized Image Deblurring . . . .

Buda Bajić, Joakim Lindblad, and Nataša Sladoje

Drawing Parrots with Charcoal. ..................

A. Alsam and H.J. Rivertz.

Unconstrained Structural Similarity-Based Optimization . . . . . . . . . . .

Daniel Otero and Edward R. Vrscay

\section{Feature Detection and Image Segmentation}

Reflectance-Based Segmentation Using Photometric and Illumination

Invariants. . . . . . . . . . . . . . . . . . . . . . . .

Jose-Antonio Pérez-Carrasco, Begoña Acha-Piñero,

Carmen Serrano-Gotarredona, and Theo Gevers

Meta-learning for Adaptive Image Segmentation . . . . . . . . . . . . .

Aymen Sellaouti, Yasmina Jaâfra, and Atef Hamouda

Dynamic Multiple View Geometry with Affine Cameras. . . . . . . . . . . . Cheng Wan, Yiquan Wu, and Jun Sato

Energy Minimization by $\alpha$-Erosion for Supervised Texture Segmentation . . . Karl Skretting and Kjersti Engan

ALOE: Augmented Local Operator for Edge Detection. . . . . . . . . . . . . . Maria De Marsico, Michele Nappi, and Daniel Riccio 


\title{
Towards a Comprehensive Evaluation of Ultrasound Speckle Reduction
}

\author{
Fernando C. Monteiro ${ }^{1(\bowtie)}$, José Rufino ${ }^{1,2}$, and Vasco Cadavez ${ }^{1,3}$ \\ 1 Polytechnic Institute of Bragança, 5301-857 Bragança, Portugal \\ monteiro@ipb.pt \\ 2 Laboratório de Instrumentação e Física Experimental de Partículas, \\ Campus de Gualtar, 4710-057 Braga, Portugal \\ 3 Mountain Research Center (CIMO), Bragança, Portugal
}

\begin{abstract}
Over the last three decades, several despeckling filters have been developed to reduce the speckle noise inherently present in ultrasound images without losing the diagnostic information. In this paper, a new intensity and feature preservation evaluation metric for full speckle reduction evaluation is proposed based contrast and feature similarities. A comparison of the despeckling methods is done, using quality metrics and visual interpretation of images profiles to evaluate their performance and show the benefits each one can contribute to noise reduction and feature preservation. To test the methods, noise-free images and simulated B-mode ultrasound images are used. This way, the despeckling techniques can be compared using numeric metrics, taking the noise-free image as a reference. In this study, a total of seventeen different speckle reduction algorithms have been documented based on adaptive filtering, diffusion filtering and wavelet filtering, with sixteen qualitative metrics estimation.
\end{abstract}

\section{Introduction}

Medical ultrasound imaging is a technique that has become more widespread than other medical imaging techniques since this technique is more accessible, less expensive, non-invasive and non-ionizing, simpler to use and produces images in real-time. However, B-mode ultrasound images are usually corrupted by the speckle artifact, which introduces fictitious structures that can not be removed by the imaging system $[11,14]$. Speckle noise is defined as multiplicative noise with a granular pattern formed due to coherent processing of backscattered signals from multiple distributed targets. Speckle degrades the quality of ultrasound images, and thus affects diagnosis. Thus, speckle reduction has become an important task in many applications with ultrasound imaging [13].

Removing noise from the original image is still a challenging research in image processing and many studies have been conducted to develop specific methods dedicated to despeckling ultrasound images $[4,13,14]$. With the rapid proliferation of despeckling filters, denoise evaluation has been becoming an important

(C) Springer International Publishing Switzerland 2014

A. Campilho and M. Kamel (Eds.): ICIAR 2014, Part I, LNCS 8814, pp. 141-149, 2014.

DOI: $10.1007 / 978-3-319-11758-4 \_16$ 
issue. A great deal of effort has been made in recent years to develop objective image quality metrics that correlate with perceived quality measurement [16,17]. Test data for evaluation includes clinical and phantom images, as well as simulated ultrasound which allow evaluation of filtering relative to an ideal speckle free reference. However, objective evaluation of noise reduction on ultrasound images is a challenging task due to the relatively low image quality.

In this study, filtered images were evaluated using several quality evaluation metrics such as average difference (AD), coefficient of correlation (CoC), gradient similarity measure (GSM), Laplacian mean square error (LMSE), maximum difference (MD), mean structural similarity index (MSSIM), the multiscale extension of MSSIM (M3SIM), normalized absolute error (NAE), normalized cross-correlation (NK), peak signal to noise ratio (PSNR), quality index based on local variance (QILV), root mean square error (RMSE), signal to noise ratio (SNR), structural content (SC) and universal quality index (UQI). All these metrics are self explanatory and hence a separate explanation for each metrics is not included due to page limitation. We also propose a new evaluation metric, the Speckle Reduction Evaluation Measure (SREM), presented in Sect 3.

The remainder of this paper is organized as follows: Sect. 2, describes the used despeckling filters. The proposed evaluation metric is explained in Sect. 3. In Sect. 4, we present the results and the discussion of the findings. Finally, conclusions are drawn in Sect. 5 .

\section{$2 \quad$ Speckle Filtering Techniques}

Speckle noise reduction has been extensively studied and many denoising algorithms have been proposed. They are classified into three groups: (i) techniques that are applied directly in the original image, (ii) techniques based on anisotropic diffusion and (iii) techniques that are applied in the wavelet domain.

Adaptive filters take a moving filter window and estimate the statistical characteristics of the image inside the filter region, such as the local mean and the local variance. Spatial adaptive filters like median, Lee [10], Frost [5] and Kuan [9] filters assume that the speckle noise is essentially a multiplicative noise. Wiener filter [7] performs smoothing of the image based on the computation of local image variance. Ideal Fourier and Butterworth filtering performs image enhancement by applying the filter function and inverse FFT on the image [11]. Bilateral filtering technique is a combination of a spatial and range filter, where each output pixel value is a Gaussian weighted average of its neighbours in both space and intensity range. This nonlinear combination of nearby pixel values, gives the well-known good performance of this filter in smoothing while preserving edges. Coup et al. [2] proposed the nonlocal means (NL-means) filter which is based on estimating each pixel intensity from the information provided from the entire image and hence it exploits the redundancy caused due to the presence of similar patterns and features in the image.

Diffusion filters remove noise from an image by modifying the image via solving a partial differential equation. Speckle reducing filters based on anisotropic 
diffusion algorithms were introduced by Perona and Malik [15] (PM-AD). Unlike conventional spatial filtering techniques, anisotropic diffusion techniques can simultaneously reduce noise and preserve image details [15]. Due to this attractive feature, many researchers have used anisotropic diffusion techniques in speckle noise reduction. Weickert [18] introduced the coherence enhancing diffusion (CED), that allows the level of smoothing to vary directionally by a tensor diffusion function. The edge enhanced anisotropic diffusion (EEAD) method is also proposed, which includes anisotropic diffusion and edge enhancement. Yu and Acton [19] first introduced partial differential equation by integrating the Lee adaptive filter and the Perona-Malik diffusion, which they called Speckle Reducing Anisotropic Diffusion (SRAD). SRAD provides significant improvement in speckle suppression and edge preservation when compared to traditional methods like Lee, Frost and Kuan filters.

Wavelet transform, unlike Fourier transform, shows localization in both time and frequency and it has proved itself to be an efficient tool for noise removal [8]. One widespread method exploited for speckle reduction is wavelet shrinkage, including VisuShrink, SureShrink [3] and BayeShrink [1]. A wavelet-based multiscale linear minimum mean square-error estimation (LMMSE) is proposed in [20], where an interscale model, the wavelet coefficients with the same spatial location across adjacent scales, was combined as a vector, to which the LMMSE in then applied.

\section{Speckle Reduction Evaluation Metric}

The speckle reduction and the preservation of edges are in general divergent. A trade-off between noise reduction and the preservation of the actual image features and contrast has to be made in order to enhance the relevant image content for diagnostic purposes. Best contrast is meant in the sense of decreasing the variance in a homogeneous region while distinct regions are well defined.

We propose a new speckle reduction evaluation metric, the SREM, that is based on the contrast and gradient similarity maps between two images. The computation of SREM index consists of two stages. In the first stage, the contrast similarity map is computed, and then in the second stage, we combine it with the gradient similarity map (GSM) to encode feature information.

Consider $f(i, j)$ as the original (noise free) image and $g(i, j)$ as the filtered image. The contrast similarity map (CSM) is defined as follow:

$$
\operatorname{CSM}(f, g)=\frac{4 \mu_{f} \mu_{g} \cdot \sigma_{f, g}}{\left(\mu_{f}^{2}+\mu_{g}^{2}+c_{1}\right) \cdot\left(\sigma_{f}^{2}+\sigma_{g}^{2}+c_{2}\right)}
$$

where $\mu$ and $\sigma$ are the mean intensity and the standard deviation of each image, $\sigma_{f, g}$ is the covariance between them, $C_{1}$ and $C_{2}$ are two constants to avoid instability when $\mu_{f}^{2}+\mu_{g}^{2}$ is very close to zero.

The gradient computation step is crucial in image processing and segmentation. Several approaches have been proposed in literature that start by convolving the image with a bank of linear filters tuned to various orientation and spatial 
frequencies [12]. These approaches were inspired by models of processing in the early stages of the primate visual system providing a simple but biologically plausible model.

In our approach, the image is first convolved with Gaussian oriented filter pairs to extract the magnitude of orientation energy (OE) of edge responses as used by Malik et al. in [12]. The filters are tuned to detect edges of different shapes, parametrized by $\rho=\left\{\rho_{o}, \rho_{s}, \rho_{e}\right\}$, where $\rho_{o}, \rho_{s}$ and $\rho_{e}$ refer to orientation, scale and elongation respectively. Given image $I$, the orientation energy approach can be used to detect and localize the composite edges, and it is defined as:

$$
O E(\rho)=\left(I * F_{e}(\rho)\right)^{2}+\left(I * F_{o}(\rho)\right)^{2}
$$

where $F_{e}(\rho)$ and $F_{o}(\rho)$ represent a quadrature pair of even and odd-symmetric filters which differ in their spatial phases. The even-phase filters are the secondorder derivative and the corresponding odd-symmetric filters are their Hilbert transforms which correspond to the first-order derivative, both smoothed with Gaussian functions specified by $\rho$.

At each pixel $i$, we can define the dominant orientation energy $\left(O E_{i}(\rho)^{*}\right)$ and the parameter $\left(\rho_{i}^{*}\right)$ as the maximum energy across scale, orientation and elongation:

$$
O E_{i}(\rho)^{*}=\max O E(\rho) \quad \rho_{i}^{*}=\arg \max O E(\rho)
$$

Gradient orientation energy $O E(\rho)$ has a maximum response for contours of shape $\rho$, whereas the zero-crossing of filter $F_{e}(\rho)$ locate the positions of the edges. The value $O E^{*}$ is kept at the location of $i$ only if it is greater than or equal to the neighbouring values. Otherwise it is replaced with a value of zero.

The gradient similarity map (GSM) between images $f(i, j)$ and $g(i, j)$ is defined as follow:

$$
G S M=\frac{2 O E_{f} \cdot O E_{g}+T_{1}}{O E_{f}+O E_{g}+T_{1}}
$$

where $T_{1}$ is a positive constant depending on the dynamic range of GSM values.

Having obtained the contrast similarity CSM and the gradient orientation similarity at each location, the overall similarity between images $f(i, j)$ and $g(i, j)$ can be calculated:

$$
S R E M=\frac{\sum C S M(i, j) \cdot G S M(i, j)}{\sum G S M(i, j)}
$$

\section{Experimental Results}

The performance of the proposed SREM will be evaluated and compared with representative state-of-the-art noise reduction evaluation metrics. In this action, the evaluation metrics are tested on seventeen despeckling filters, with simulated ultrasound images. 


\subsection{Field II Speckle Noise Images}

To evaluate the denoising filters it is necessary to have reference images (without noise or with low level of noise) used to measure the improvement in image quality. Ideally both noisy and reference images must be obtained with the same ecograph and under the same conditions. However, due to the highly operator dependence of the ultrasound exams and the random variation of scattering it is useful to use synthetic images obtained, e.g., by means of computer simulations.

We used the Field II [6] to simulate the ultrasound images. This program assumes that the pressure field has linear propagation and is able to calculate the pulsed and continuous pressure field for different transducers. Depending on the number of points and transducer frequency, each image simulation can take several hours when executed sequentially. In order to decrease the simulation times, we have decided to parallelize its execution.

It was concluded from the code that the image lines were processed without any data dependencies; moreover, the code was already prepared to support the processing of the same image by several application instances, by skipping a line if its specific result file was detected in the file system; the code was then modified in order to accept the necessary parameters to process a line interval, instead of the full set of lines. In this way, different line intervals could be assigned to different CPU cores; this assignment was conducted by a set of wrapper scripts, developed in BASH, that generated jobs submitted to the job manager of a Linux HPC cluster; a separate queue was created for the simulations, with 5 nodes, each one with an Intel Core i7 4770 quad-core CPU, for a total of 20 cores.

The simulated images were generated using three levels of point scatterers randomly distributed within the field, $5 \times 10^{5}, 1 \times 10^{6}$ and $2 \times 10^{6}$ points, and transducer frequencies of 3,5 and $7 \mathrm{MHz}$. These parameters produce different levels of speckle noise as shown in Fig. 1.
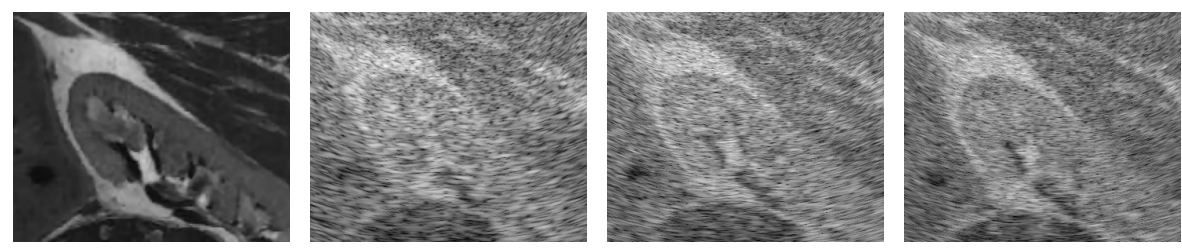

Fig. 1. Speckle simulations with Field II. (a) Reference image. (b) Speckle with $5 \times 10^{5}$ points and $3 \mathrm{MHz}$. (c) Speckle with $10^{6}$ points and $5 \mathrm{MHz}$. (d) Speckle with $2 \times 10^{6}$ points and $7 \mathrm{MHz}$.

\subsection{Example to Demonstrate the Effectiveness of SREM}

Figure 2 shows an example to demonstrate the effectiveness of SREM in evaluating the perceptible speckle reduction. Figure 2(a) is the simulated noisy image, with $10^{6}$ points and transducer frequency of $7 \mathrm{MHz}$. Figures 2(b)-(d) show three filtered images with different despeckled levels. 

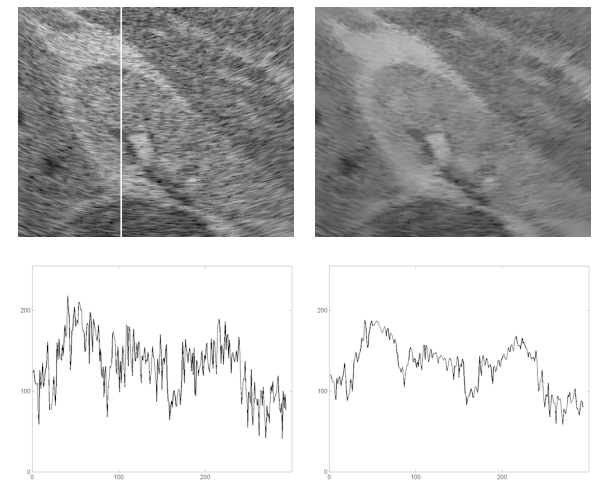

(a)

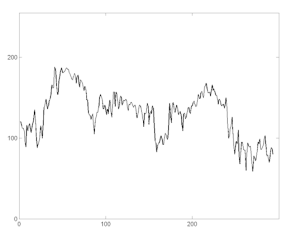

(b)
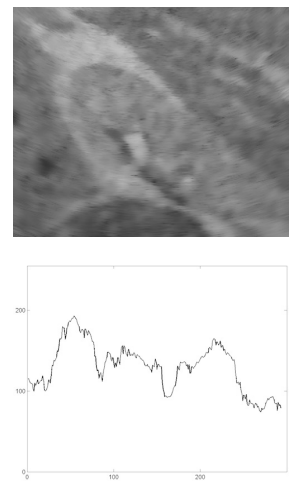

(c)
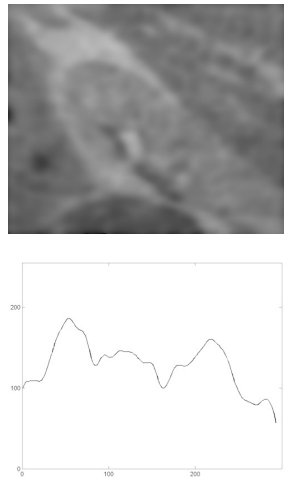

(d)

Fig. 2. Profile extracted from the white column before and after denoising. (a) Noisy image. (b) Kuan Filter. (c) Bilateral filter. (d) PM-AD filter.

The speckle evaluation of Fig. 2, with reference image from Fig. 1(a), are summarized in Table 1, where a higher score mean higher image quality.

Table 1. Quality evaluation of images in Fig.2

\begin{tabular}{lcccc}
\hline Metric & Fig. 2(a) & Fig. 2(b) & Fig. 2(c) & Fig. 2(d) \\
\hline SREM & 0.129 & 0.456 & 0.679 & 0.760 \\
SNR & 6.938 & 7.257 & 7.277 & 7.386 \\
UQI & 0.025 & 0.046 & 0.068 & 0.090 \\
CoC & 0.458 & 0.587 & 0.623 & 0.644 \\
GSM & 0.928 & 0.964 & 0.972 & 0.972 \\
MSSIM & 0.124 & 0.338 & 0.449 & 0.520 \\
\hline
\end{tabular}

From the profiles of Fig.2 and from the scores in Table 1, we can conclude that the quality scores computed by SREM correlate with the other evaluation metric. Even more, the SREM produces a higher variation over the different results, only followed by UQI and MSSIM.

\subsection{Overall Performance Comparison}

We apply despeckling filters over the simulated images and evaluate the results with the evaluation metrics. The average of the results obtained with each metric is presented in Table 2. The arrow under each metric indicates the expected measure tendency for the best despeckling filters. The Pearson linear correlation coefficient (PCC) is also presented on the table. 
From the analysis of PCC we can see that most of the metrics have a low variation in their evaluations. The exception are the LMSE, MMSIM, UQI, QILV and SREM. However, as LMSE quantifies only the average distortion in edge pixel locations between each filtered image it does not evaluate the speckle reduction inside the regions.

Table 2. Ranking of despeckling filters according to their performance computed by SREM and state-of-the-art evaluation metrics

\begin{tabular}{|c|c|c|c|c|c|c|c|c|c|c|c|c|c|c|c|c|}
\hline Filters & RMSE & MD & AD & NAE & $\mathrm{SC}$ & SNR & PSNR & LMSE & UQI & NK & $\mathrm{CoC}$ & MSSIM & M3SIM & QILV & GSM & SREM \\
\hline & $\downarrow$ & $\downarrow$ & $\downarrow$ & $\downarrow$ & $\downarrow$ & $\uparrow$ & $\uparrow$ & $\uparrow$ & $\uparrow$ & $\uparrow$ & $\uparrow$ & $\uparrow$ & $\uparrow$ & $\uparrow$ & $\uparrow$ & $\uparrow$ \\
\hline PM-AD & 69.19 & 141.78 & 62.22 & 0.96 & 0.34 & 6.84 & 11.37 & 1.06 & 0.06 & 1.56 & 0.59 & 0.49 & 0.23 & 0.01 & 0.97 & 0.72 \\
\hline NL-means & 72.61 & 152.00 & 65.88 & 1.01 & 0.32 & 6.59 & 10.94 & 1.25 & 0.06 & 1.60 & 0.58 & 0.46 & 0.23 & 0.02 & 0.97 & 0.69 \\
\hline Frost & 70.03 & 148.33 & 62.85 & 0.97 & 0.34 & 6.77 & 11.26 & 1.10 & 0.06 & 1.57 & 0.57 & 0.45 & 0.22 & 0.02 & 0.97 & 0.69 \\
\hline Median & 71.08 & 151.22 & 63.86 & 0.99 & 0.33 & 6.70 & 11.13 & 1.86 & 0.06 & 1.58 & 0.56 & 0.45 & 0.22 & 0.03 & 0.97 & 0.67 \\
\hline EEAD & 69.08 & 162.00 & 61.19 & 0.95 & 0.34 & 6.83 & 11.38 & 20.46 & 0.06 & 1.55 & 0.54 & 0.46 & 0.23 & 0.04 & 0.97 & 0.67 \\
\hline Bayes & 70.30 & 158.11 & 62.90 & 0.97 & 0.34 & 6.75 & 11.23 & 1.07 & 0.05 & 1.57 & 0.56 & 0.44 & 0.22 & 0.04 & 0.97 & 0.66 \\
\hline Sure & 70.30 & 158.11 & 62.90 & 0.97 & 0.34 & 6.75 & 11.23 & 1.07 & 0.05 & 1.57 & 0.56 & 0.44 & 0.22 & 0.04 & 0.97 & 0.66 \\
\hline Wiener & 70.05 & 163.78 & 62.54 & 0.97 & 0.34 & 6.76 & 11.26 & 5.54 & 0.05 & 1.57 & 0.55 & 0.42 & 0.21 & 0.06 & 0.97 & 0.62 \\
\hline Bilateral & 70.69 & 151.67 & 63.55 & 0.98 & 0.33 & 6.72 & 11.18 & 3.47 & 0.05 & 1.58 & 0.57 & 0.41 & 0.22 & 0.05 & 0.97 & 0.62 \\
\hline Fourier & 70.58 & 174.22 & 62.69 & 0.97 & 0.34 & 6.71 & 11.19 & 1.15 & 0.05 & 1.57 & 0.53 & 0.41 & 0.20 & 0.05 & 0.97 & 0.60 \\
\hline LMMSE & 70.68 & 162.11 & 62.91 & 0.97 & 0.34 & 6.71 & 11.18 & 1.16 & 0.05 & 1.57 & 0.54 & 0.38 & 0.20 & 0.07 & 0.97 & 0.57 \\
\hline Butter & 70.86 & 179.22 & 62.09 & 0.96 & 0.32 & 6.78 & 11.15 & 1.83 & 0.05 & 1.62 & 0.59 & 0.37 & 0.24 & 0.12 & 0.97 & 0.54 \\
\hline CED & 70.55 & 155.78 & 62.92 & 0.97 & 0.34 & 6.72 & 11.20 & 3.28 & 0.04 & 1.57 & 0.54 & 0.32 & 0.19 & 0.06 & 0.96 & 0.44 \\
\hline Lee & 70.35 & 156.44 & 62.75 & 0.97 & 0.34 & 6.73 & 11.22 & 5.44 & 0.04 & 1.57 & 0.54 & 0.33 & 0.20 & 0.09 & 0.96 & 0.44 \\
\hline Visu & 70.74 & 167.00 & 62.93 & 0.98 & 0.34 & 6.70 & 11.17 & 4.30 & 0.04 & 1.57 & 0.53 & 0.30 & 0.19 & 0.08 & 0.96 & 0.41 \\
\hline Kuan & 70.47 & 157.56 & 62.78 & 0.97 & 0.34 & 6.72 & 11.21 & 6.11 & 0.04 & 1.57 & 0.54 & 0.31 & 0.19 & 0.09 & 0.96 & 0.41 \\
\hline SRAD & 74.30 & 176.11 & 66.31 & 1.03 & 0.32 & 6.45 & 10.74 & 15.79 & 0.04 & 1.61 & 0.51 & 0.29 & 0.18 & 0.08 & 0.95 & 0.37 \\
\hline Noisy & 73.60 & 189.33 & 62.91 & 0.99 & 0.33 & 6.44 & 10.82 & 59.55 & 0.02 & 1.57 & 0.42 & 0.11 & 0.14 & 0.02 & 0.92 & 0.12 \\
\hline $\mathrm{PCC}$ & 1.91 & 7.44 & 1.93 & 1.76 & 2.09 & 1.62 & 1.49 & 186.46 & 23.24 & 1.11 & 6.91 & 23.94 & 11.21 & 56.60 & 1.18 & 28.41 \\
\hline
\end{tabular}

To test the effectiveness of detail preservation of the despeckling filters, we compared the despeckled images and the profile extracted from an image column before and after denoising, as shown in Fig. 3, for a transducer frequency of 5 $\mathrm{MHz}$. From the profiles analysis, we find that almost every methods reduced the speckle noise in homogeneous regions. The intensity variation caused by speckle is still obvious in the images filtered by Kuan, CED and VisuShrink filters. The visual analysis indicates that the best despeckling filters are PMAD, NL-means and Frost. The SRAD, Kuan and VisuShrink filters exhibit poor performance results. This analysis correlates well with the SREM evaluation results.

\section{Conclusion}

In this paper, a new evaluation metric, namely SREM, is proposed based on contrast similarity map and edge preservation which correlates well with other evaluation metrics. The underlying principle of SREM is that humans distinguish an image mainly based on its salient low-level features. The SREM uses contrast and gradient maps to represent complementary aspects of the image visual quality. In this study, a total of eighteen different speckle reduction algorithms have been documented based on spatial filtering, diffusion filtering and wavelet filtering, with seventeen quantitative metrics estimation. 

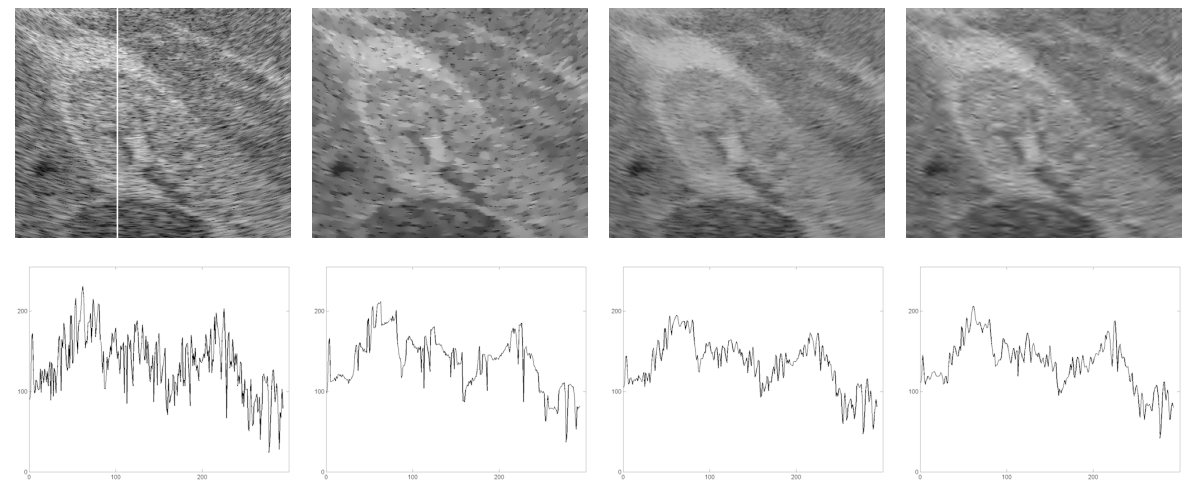

SRAD

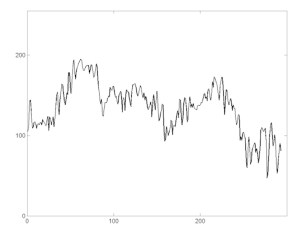

Kuan

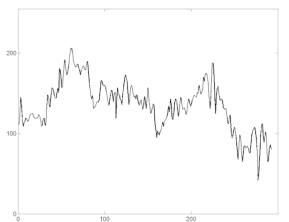

Noisy
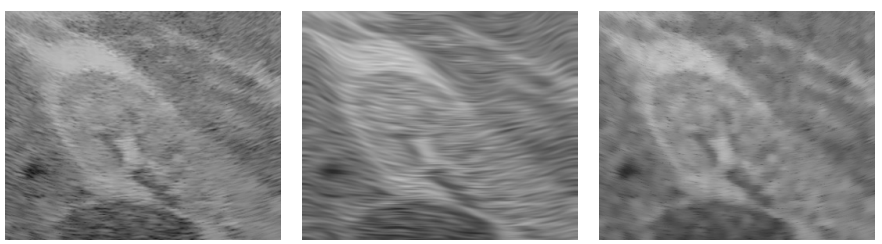

Visushrink
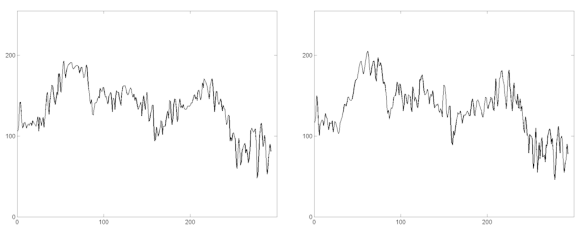

Lee

CED

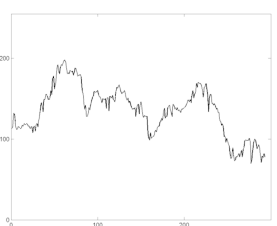

Bilateral
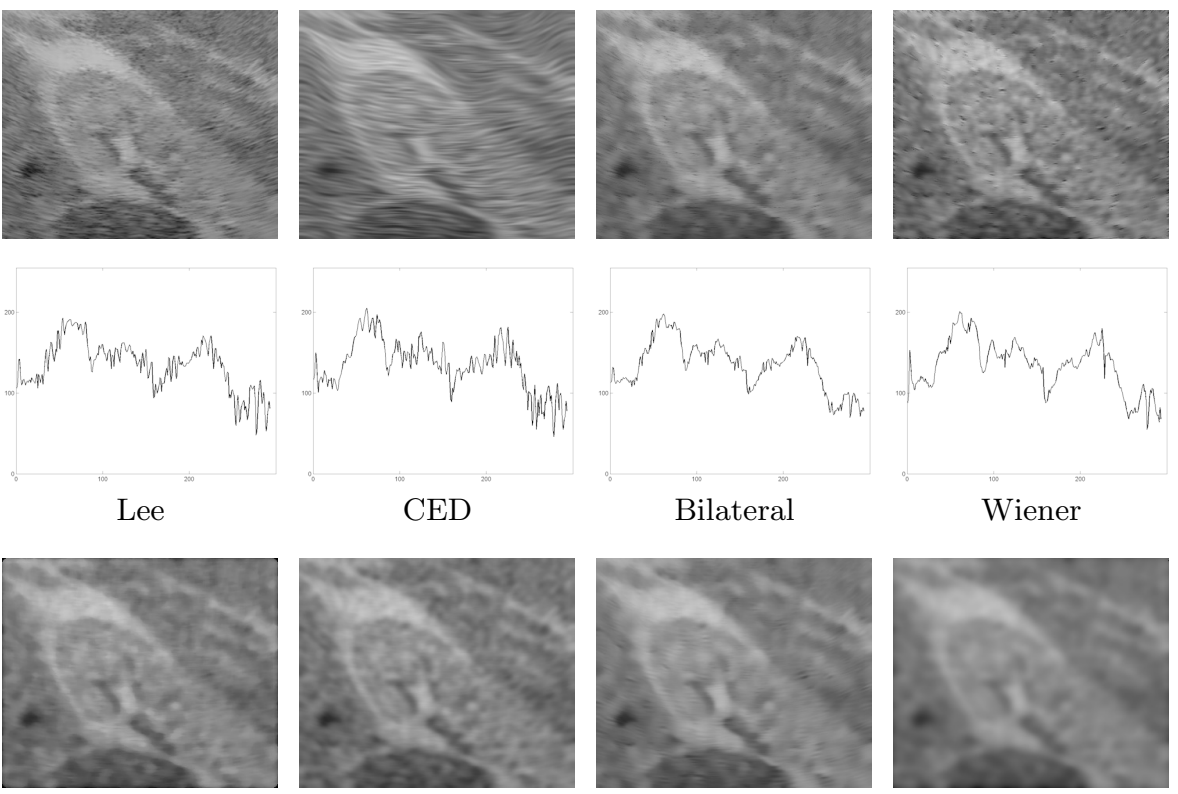

Wiener

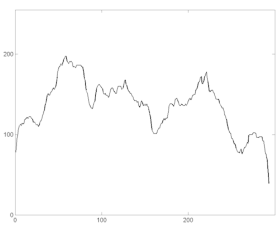

Median

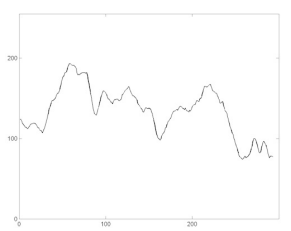

Frost

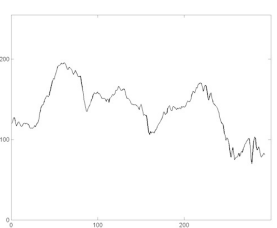

NL-means
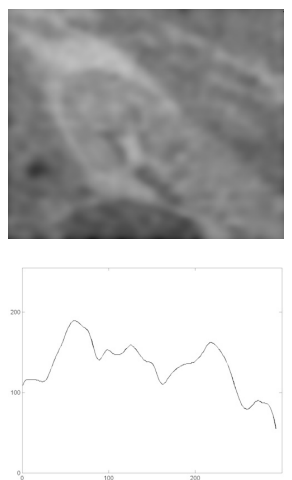

PM-AD

Fig. 3. Despeckled images and profiles extracted from the white column before and after denoising in simulated images with $10^{6}$ points and $5 \mathrm{MHz}$ transducer 


\section{References}

1. Chang, S., Yu, B., Vetterli, M.: Adaptive wavelet thresholding for image denoising and compression. IEEE Trans. Image Processing 9(9), 1532-1546 (2000)

2. Coupé, P., Hellier, P., Kervrann, C., Barillot, C.: Nonlocal means-based speckle filtering for ultrasound images. IEEE Transactions on Image Processing 18(10), 2221-2229 (2009)

3. Donoho, D.L., Johnstone, I.M.: Adapting to unknown smoothness via wavelet shrinkage. Journal of American Statistical Association 90(432), 1200-1224 (1995)

4. Finn, S., Glavin, M., Jones, E.: Echocardiographic speckle reduction comparison. IEEE Trans. Ultrasonics, Ferroelectrics Freq. Control 58(1), 82-101 (2011)

5. Frost, V., Stiles, J., Shanmugan, K., Holtzman, J.: A model for radar images and its application to adaptive digital filtering of multiplicative noise. IEEE Transactions on Pattern Analysis and Machine Intelligence 4(2), 157-166 (1982)

6. Jensen, J.: Simulation of advanced ultrasound systems using field ii. In: International Symposium on Biomedical Imaging: Nano to Macro, pp. 636-639 (2004)

7. Jin, F., Fieguth, P., Winger, L., Jernigan, E.: Adaptive wiener filtering of noisy images and image sequences. In: Proceedings of International Conference on Image Processing, vol. 3, p. III-349 (2003)

8. Khare, A., Khare, M., Jeong, Y., Kim, H., Jeon, M.: Despeckling of medical ultrasound images using daubechies complex wavelet transform. Signal Processing 90(2), 428-439 (2010)

9. Kuan, D., Sawchuk, A., Strand, T., Chavel, P.: Adaptive noise smoothing filter for images with signal-dependent noise. IEEE Transactions on Pattern Analysis and Machine Intelligence 7(2), 165-177 (1985)

10. Lee, J.-S.: Digital image enhancement and noise filtering by use of local statistics. IEEE Trans. on Pattern Analysis and Machine Intelligence 2(2), 165-168 (1980)

11. Loizou, C., Pattichis, C.: Despeckle filtering algorithms and software for ultrasound imaging. Synthesis Lect. Algorithms Soft. Engineering 1(1), 1-166 (2008)

12. Malik, J., Belongie, S., Leung, T., Shi, J.: Contour and texture analysis for image segmentation. International Journal of Computer Vision 43(1), 7-27 (2001)

13. Mateo, J.L., Fernández-Caballero, A.: Finding out general tendencies in speckle noise reduction in ultrasound images. Expert Systems with Applications 36(4), 7786-7797 (2009)

14. Ortiz, S., Chiu, T., Fox, M.D.: Ultrasound image enhancement: A review. Biomedical Signal Processing and Control 7(5), 419-428 (2012)

15. Perona, P., Malik, J.: Scale-space and edge detection using anisotropic diffusion. IEEE Trans. on Pattern Analysis and Machine Intelligence 12(7), 629-639 (1990)

16. Rosa, R., Monteiro, F.C.: Speckle ultrasound image filtering: Performance analysis and comparison. In: Computational Vision and Medical Image Processing: VIPIMAGE 2013, pp. 65-70 (2013)

17. Wang, Z., Li, Q.: Information content weighting for perceptual image quality assessment. IEEE Transactions on Image Processing 20(5), 1185-1198 (2011)

18. Weickert, J.: Coherence-enhancing diffusion filtering. International Journal of Computer Vision 31(2-3), 111-127 (1999)

19. Yu, Y., Acton, S.T.: Speckle reducing anisotropic diffusion. IEEE Transactions on Image Processing 11(11), 1260-1270 (2002)

20. Zhang, D., Bao, P., Wu, X.: Multiscale lmmse-based image denoising with optimal wavelet selection. IEEE Transactions on Circuits and Systems for Video Technology 15(4), 469-481 (2005) 